\title{
Henri Nonn, 1999, Villes et aménagement régional en
} Alsace

Collection Société, Les Études de La Documentation Française, La Documentation Française, Paris, 246 p.

\section{Gabriel Wackermann}

\section{CpenEdition} Journals

Édition électronique

URL : http://journals.openedition.org/rge/4376

DOI : $10.4000 /$ rge.4376

ISSN : 2108-6478

Éditeur

Association des géographes de l'Est

Édition imprimée

Date de publication : 1 septembre 1999

ISSN : 0035-3213

Référence électronique

Gabriel Wackermann, « Henri Nonn, 1999, Villes et aménagement régional en Alsace », Revue

Géographique de l'Est [En ligne], vol. 39 / 4 | 1999, mis en ligne le 03 septembre 2013, consulté le 25 septembre 2020. URL : http://journals.openedition.org/rge/4376 ; DOI : https://doi.org/10.4000/rge. 4376

Ce document a été généré automatiquement le 25 septembre 2020

Tous droits réservés 


\section{Henri Nonn, 1999, Villes et aménagement régional en Alsace}

Collection Société, Les Études de La Documentation Française, La Documentation Française, Paris, 246 p.

\section{Gabriel Wackermann}

\section{RÉFÉRENCE}

Henri Nonn, 1999, Villes et aménagement régional en Alsace, Collection Société, Les Études de La Documentation Française, La Documentation Française, Paris, 246 p.

\section{La dynamique urbaine, force d'entraînement inachevée de l'Alsace}

1 La présentation en quatrième de couverture du dernier ouvrage de Henri Nonn comme un panorama exhaustif du système urbain alsacien n'est en aucune façon surfaite, bien au contraire. L'auteur, grand spécialiste des questions urbaines contemporaines et des problèmes d'aménagement, à la fois en tant qu'universitaire et collaborateur récent de la DATAR qui l'a chargé de la mission d'animateur interrégional de la prospective relative au Grand Est, a réussi en un livre d'une très riche densité à faire comprendre au lecteur professionnel ainsi qu'au grand public cultivé l'essentiel du fonctionnement socio-économique et des perspectives de l'Alsace. Cette parution se situe avec bonheur dans le prolongement du volume, fondamental lui aussi, composé par le même auteur, en collaboration avec Jean-Alain Héraud, sur « Les économies industrielles en France de l'Est: tissus et réseaux en évolution ", paru en 1995 aux Presses universitaires de Strasbourg, dans les Publications de la Maison des Sciences de l'Homme (305 p.).

2 Le plan adopté donne le ton et témoigne de l'originalité de la démarche. L'auteur articule ses analyses et réflexions autour de trois grandes approches: la métropolisation en Alsace, l'organisation urbaine régionale, les villes et réseaux « par 
delà l'espace régional ». Ainsi l'Alsace apparaît à la fois dans ses structures internes et son degré d'insertion à l'espace ambiant, autant que dans son aptitude à l'ouverture vers l'extérieur. Les observations auxquelles se livre Henri Nonn nous révèlent les multiples singularités sous-régionales et locales, livrant implicitement une Alsace plurielle qui tranche avec une certaine imagerie d'Épinal relative à l'unité, voire l'unicité d'une région faite, malgré tout, elle aussi, de pièces et de morceaux que l'Histoire et le présent ont soudés ensemble aussi bien que possible. Le rôle des aires périphériques, particulièrement perceptible à travers les villes, est mis en relief: Mulhouse et la Regio se singularisent notamment, mais aussi les petites villes du nord, voire les têtes de pont rhénanes parmi lesquelles Colmar se détache aisément.

3 Si la construction urbaine alsacienne s'est effectuée de façon permanente dans les deux sens, du bas vers le haut de la pyramide et inversement, la métropolisation s'impose désormais comme force d'entraînement capitale. Strasbourg est présentée comme une eurocité. Cette position « relativement nouvelle » « reste un défi permanent qui appelle une veille stratégique et prospective de tous les instants, puisque située dans un champ de concurrence sévère, où les retards pris deviennent aussitôt des handicaps ». La force urbaine acquise continue toutefois à peser sur ce mouvement de métropolisation générale, qui se nourrit constamment de la vitalité des réseaux de petites et moyennes villes. Toute politique d'aménagement qui veut bénéficier d'un minimum de succès est tenue de s'appuyer sur la diversité du tissu urbain et les spécificités identitaires. Plus le niveau urbain est proche du monde rural, plus il est important comme point d'appui pour le développement.

4 La précision des propos n'empêche pas l'auteur d'être tout en nuance. Le constat est rigoureux et livre les bases d'une construction spatiale - régionale, rhénane et européenne -, efficace, si toutefois les responsables de l'aménagement du territoire veulent bien s'en inspirer. Il relève les nombreux atouts de l'Alsace, les efforts d'intégration réalisés en vue d'une meilleure insertion de cette région au tissu national, aux exigences européennes et mondiales. Il révèle l'importance de l'Histoire, la richesse urbaine d'un espace multicéphale évoluant insensiblement vers une région urbaine. Dès à présent la ville est déterminante pour l'évolution générale ; les milieux ruraux, déjà fortement urbanisés, laissant fort peu de place à la campagne profonde, sont tributaires du degré de santé et de dynamisme urbains. La notion de hiérarchie urbaine, pendant longtemps considérée comme essentielle, est heureusement relativisée au bénéfice de la complémentarité, de la structure en réseaux, du système de développement. Henri Nonn insiste sur le fait que celui-ci « articule mieux que par le passé ses sous-systèmes économique, scientifique et technologique et institutionnel, en intégrant le rôle important des équipements d'accueil et de la culture, ainsi qu'une politique suivie à leur sujet ».

5 L'auteur est néanmoins sans complaisance avec une région dont les vraies potentialités sont loin d'être épuisées. Il insiste sur les opportunités à ne pas manquer : la position européenne croissante, qui exigerait cependant une meilleure intégration des préoccupations sociales et politiques aux succès économiques; une valorisation régionale renforcée de l'ancrage national continental; la poursuite de la mise en réseaux aux diverses échelles spatiales; le renforcement de la recherche qualitative du développement. Il met en garde contre la "menace» de dépendance qui pèse sur certaines villes alsaciennes insuffisamment parées contre les effets de concurrence «jouant des flexibilités,... des rapports de force». Il regrette que les lenteurs 
inhérentes aux mesures prises aux échelles nationales et européennes pénalisent les projets et actions du long terme. Il constate que le tarissement général des ressources publiques réduit la réalisation des justes ambitions de promotion régionale.

6 À propos des occasions perdues au moment des années de vaches grasses, je ne peux m'empêcher de rappeler la frilosité, voire la profonde méfiance avec laquelle, dans les années 1950 et surtout 1960, les rapports avec nos partenaires allemands ont été freinés ; à Strasbourg même l'initiative de la Regio, prise par les responsables hautrhinois en direction des collectivités publiques voisines, suisses et allemandes, a été jugée avec beaucoup de réticence, une attitude qui n'a fait que renforcer les tendances centrifuges d'une région dont la cohésion autour des vrais problèmes d'avenir fit encore bien défaut ; contrairement à la version officielle, le "glacis » frontalier a duré bien au-delà des dissensions franco-allemandes; il a été entretenu par les principaux élus politiques alsaciens eux-mêmes, en dépit de leur profession de foi européenne. Les retards accumulés rendent ainsi les solutions plus difficiles, au moment où la manne publique se fait plus parcimonieuse.

7 Aussi Henri Nonn relève-t-il que les récentes fractures socio-spatiales fragilisent les acquis de toute nature et suscitent des faiblesses structurelles jusqu'au niveau intraurbain, mettant en cause la solidarité citoyenne, à tel point que l'ensemble des atouts du système urbain alsacien est mis à rude épreuve. La politique communautaire de recomposition spatiale provoque de surcroît « des interrogations sur le devenir des territoires situés aux marches ou aux interfaces, ou (et) sur des axes anciens que la reconfiguration esquissée paraît "cisailler". On songe ici à l'axe rhénan millénaire qui, au niveau du Rhin supérieur, se trouverait écartelé entre un ensemble du "centre des capitales" rassemblant les principaux pôles politiques, économiques et financiers sur le nord-ouest de l'Europe et un ensemble dit "arc alpin" où se regroupent les aires technologiques majeures ».

8 Autant de thèmes de réflexion qui requièrent une vigilance générale et une action politique régionale vigoureuse. L'Alsace demeure un espace français original, qui n'est pas aussi rhénan que les autres riverains de ce fleuve, et qui continue à mériter une attention spécifique dans le cadre d'une évolution dont elle est partie prenante à part entière. L'excellent livre de Henri Nonn, figures et tableaux pertinents à l'appui, par la démarche méthodique adoptée par l'auteur, sachant passer, selon le cas, du général au particulier et inversement, n'est pas uniquement un ouvrage de référence, mais aussi une source de méditation sur le devenir d'une région complexe et sans doute encore féconde en avenir. 